\title{
Small-Scale Surface Flows and their Implications for Solar Activity
}

\author{
Marc L. DeRosa \\ Lockheed Martin Solar and Astrophysics Laboratory \\ 3251 Hanover St., O/ADBS B/252, Palo Alto, CA 94304, USA \\ email: derosa@lmsal.com
}

\begin{abstract}
The broad range of dynamics exhibited by plasma motions within the solar interior affects many aspects of the generation and transport of magnetic fields during the solar magnetic activity cycle. On the photosphere, such dynamics include the differential rotation, meridional flows, and a hierarchy of convection cells, and these fluid motions are observed to readily advect any small-scale magnetic fields embedded within them. While the effects of large-scale flows on the global activity cycle are well known, it is becoming increasingly apparent that smallscale dynamics can also affect global magnetic activity throughout the solar cycle. Such effects include variations in the strength of the magnetic dipole moment with time, and the timing of the reversals of the polar-cap flux. In this article, several aspects of this coupling between small and large scales will be illustrated, and the implications of such coupling on the solar activity cycle will be discussed.
\end{abstract}

Keywords. Sun: photosphere, Sun: activity

\section{Introduction}

The sun undergoes cyclic periods of intense magnetism, exhibited by the variable number of bipolar magnetic flux concentrations that emerge onto the solar photosphere over the course of each 11-year sunspot cycle. These emergent bipoles possess a broad range of sizes and fluxes, ranging from large active regions that span several tens of $\mathrm{Mm}$ and contain of order $10^{23} \mathrm{Mx}$, to small, point-like ephemeral concentrations having fluxes of order $10^{18} \mathrm{Mx}$ and separated by mere hundreds of $\mathrm{km}$.

The origin of these photospheric magnetic fields, occurring via processes commonly referred to as the solar dynamo, rests with the highly turbulent convection and strong shear layers below the surface that serve to stretch, twist, and otherwise deform the magnetic fields permeating the solar interior. The relative buoyancy of magnetized plasma (compared with surrounding nonmagnetized plasma) within the convection zone causes some of this magnetic field to rise upward and penetrate the photosphere where it can be observed. Yet, because direct observations of the solar interior are unavailable, our knowledge of the solar dynamo is based largely on inferences from observations of surface features, and as a result the development of a solid understanding of the inner workings of the solar dynamo is sometimes challenging.

Once magnetic field appears on the photosphere, all but the strongest fields are observed to evolve in response to organized fluid motions. Global differential rotation causes surface flux patterns to become gradually sheared, while persistent meridional motions slowly advect flux poleward. Additionally, small-scale but fast convective outflows associated with supergranulation and granulation serve to confine small-scale flux concentrations within the intercellular lanes and disperse this flux in a random-walk-like fashion. Together, all of these flows transport flux across the photosphere, and in so doing enable 
this flux to self-interact. Such interactions between like polarities create larger flux concentrations, while collisions of opposite polarities flux result in the removal of flux from the surface.

On the largest scales, flux emergence onto the photosphere occurs in a highly ordered fashion, with well-defined trends governing the latitude, polarity, and bipole axis tilt angle (with respect to the equator) of emergent bipolar flux. These systematic tilt angles, coupled with the latitudinal transport and convective dispersal of flux, cause an excess of magnetic field of one polarity to accumulate at each pole throughout the course of each cycle. These polar caps of flux exist until the following cycle, where this unipolar field is cancelled by and replaced with polar-cap fields of the opposite polarity. We can thus infer that the strength of the resulting global dipole depends on the amount of flux that emerges throughout each sunspot cycle, as well as on other factors such as the rate of cancellation of flux and on the rate at which the field is transported poleward by organized meridional flows.

This article focuses on the effects that the small-scale, or ephemeral, flux concentrations have on the evolution of the the larger-scale magnetic field patterns on the photosphere. First we shall review some observations of the small-scale flux elements in $\S 2$. We then use a flux-transport model in $\S 3$ to explore some of the consequences of these observations. Concluding remarks regarding the effects of these ephemeral flux elements on the solar dynamo are presented in $\S 4$.

\section{Properties of Small-Scale Flux Elements}

The variable activity on the sun is most evident in the increase and decrease in the emergence rate of sunspots and active regions throughout each cycle. (Active regions typically have absolute fluxes of order $10^{20} \mathrm{Mx}$ or more.) We define solar maximum to be the period when the emergence rate of sunspots and active regions is greatest; conversely, solar minimum sees very few active regions emerge onto the photosphere. The period between successive solar minima (i.e., one sunspot cycle) is approximately 11 years, but has been observed to vary between 9 and 13 years.

Complementing the large-scale bipolar flux of sunspots and active regions is an abundant population of smaller flux concentrations. Despite their smaller size, this small-scale magnetism has an aggregate unsigned flux that adds up to be much greater than the total flux in sunspots and active regions. Interestingly, measurements of bipole emergence rates and tilts by Harvey (1993) during Cycle 21, and by Hagenaar et al. (2003) and Muenier (2003) during Cycle 23, indicate that smaller-scale bipoles with absolute fluxes below $10^{20} \mathrm{Mx}$ also seem to possess the same cycle-dependent trends as the sunspot and active region bipoles, but to a lesser degree. The results of Hagenaar et al. (2003) suggest that the cycle variation only applies to bipoles having fluxes greater than $5 \times 10^{19} \mathrm{Mx}$; below this level, they find that the number of ephemeral regions at the extreme smallest end of the scale either show almost no variation in time or show a possible weak variation in antiphase with the cycle, a result also suggested by counts of network bright points at different phases of the solar cycle (e.g., Foukal et al. 1991 and Muller \& Roudier 1994).

Both Harvey (1993) and Hagenaar et al. (2003) determine the orientation of the bipole tilt axes of all ephemeral regions in their respective samples. $\dagger$ As with the rate of emergence, the regions possessing fluxes in the $5-30 \times 10^{19} \mathrm{Mx}$ range are statistically more likely to have the same alignment of leading and trailing poles as their larger-scale,

$\dagger$ The tilt axis of a bipole is usually defined as the angle between the line connecting the two polarities of the bipole and the equator of the sun. 
active-region counterparts, albeit with more scatter in the distribution functions. However, when considering the smallest observable bipoles (having absolute fluxes less than $5 \times 10^{19} \mathrm{Mx}$ ), this trend apparently disappears, as these bipoles appear to be oriented randomly.

Another property of ephemeral flux concentrations that affects the dispersal across the photosphere is their mobility. Small-scale magnetic elements are observed to be more mobile than large-scale concentrations (Schrijver et al. 1996; Hagenaar et al. 1999), resulting in an enhancement in the dispersal rate of these concentrations relative to active region flux. This effect is most likely caused by a coupling between the convective fluid motions and the magnetism, due to the tendency for stronger magnetic concentrations to inhibit fluid motions while weaker magnetic concentrations seem to be passively advected by surrounding flows.

Properties of small-scale flux such as described here are likely to affect the evolution of surface flux. Without their presence, the patterns resulting from the decay of large-scale and active-region-sized flux concentrations would be very different. It is also intriguing that ephemeral regions with fluxes well below typical active-region thresholds possess statistically significant preferences for their emergence rates and orientations, raising questions of their origin and of their importance in the evolution of the large-scale surface magnetic field. Our aim in this article is to examine the impact that small-scale flux might have on the global-scale dynamics, by using a surface-flux transport model to simulate the evolution and transport of a large population of surface-flux elements.

\section{Flux-Transport Simulations of Surface Activity}

We here use surface-flux transport models to investigate the importance of the population of ephemeral flux elements on the formation and evolution of large-scale flux patterns. Such models have been shown to capture fairly well the evolution of photospheric magnetic flux, and enable the study of complex, nonlinear processes that are thought to govern the dynamics of the photospheric magnetoconvection on the sun. These models also have the advantage that they are fast, and many activity cycles can be simulated in a short period of time (in contrast to models that solve the full nonlinear, threedimensional equations of magnetohydrodynamics). The surface-flux transport approach involves simulating the insertion, movement, and removal of radial flux as it evolves on a two-dimensional spherical shell representing the photosphere.

Flux-transport dynamo models were essentially formulated by Leighton (1969), who showed that poleward transport of flux naturally occurs when tilted sunspot and active region bipoles are subject to diffusion. The addition of a solar-like differential rotation profile to the model, and especially the addition of a slow poleward meridional circulation (DeVore et al. 1984; Sheeley et al. 1987), improved the agreement between the patterns of flux dispersal predicted by the models and by the patterns observed on the sun. The primary improvement was in the appearance of "streaks" of flux observed to extend poleward from the decaying active regions (sometimes also referred to as the "rush to the poles").

Here, we use the surface-flux transport model developed by Schrijver (2001), in which surface flux is represented by discrete, point-like elements that can traverse the twodimensional model photosphere (instead of as a continuous function discretized onto a grid, as used by DeVore et al. 1984 and Sheeley et al. 1987). Pairs of flux elements are inserted as bipoles onto the model photosphere according to empirical statistical distribution functions describing their emergence rates, latitudes, and bipole tilt axes. Once on the surface, flux elements move independently in response to the bulk motions associated 


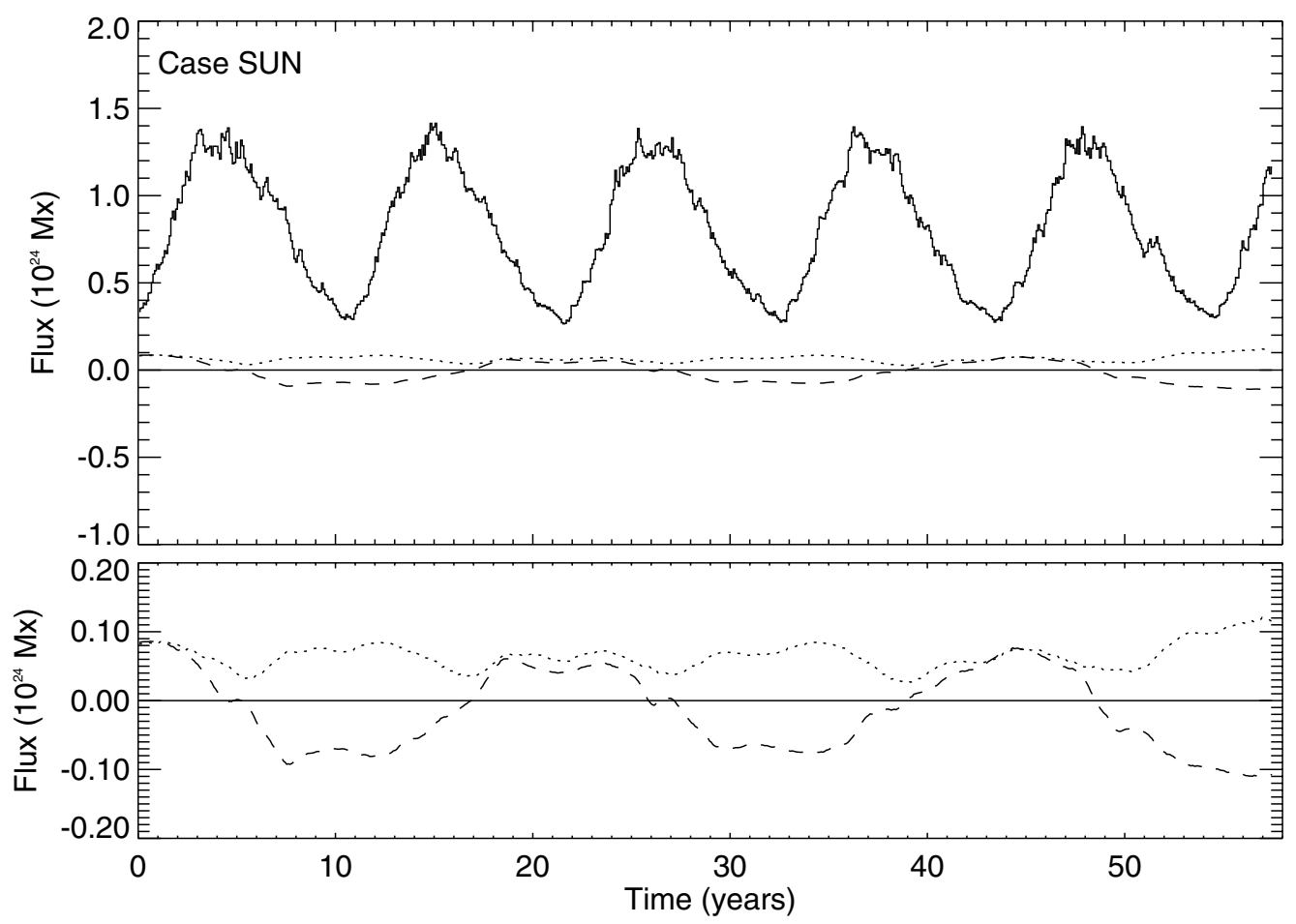

Figure 1. The total unsigned flux (solid line), total unsigned flux in the north polar cap (dotted line), and twice the net unsigned polar flux in the north polar cap (dashed line) for Case SUN. The north polar cap fluxes are evaluated using all flux north of $+60^{\circ}$ in latitude.

with differential rotation and poleward meridional flows. In the Schrijver (2001) model, these bulk flow profiles are based on time-averaged flows measured by Komm et al. (1993a) and Komm et al. (1993b) that apply to magnetic elements on the photosphere.

The convective dispersal of flux is approximated as a random walk, in which each flux element is moved some distance in a random direction during each time step. Because small-scale flux concentrations on the sun are more mobile than larger concentrations, as found in Schrijver et al. (1996), the mean free path of flux elements in the model is set to be dependent on the flux contained in each element.

As the flux elements get shuffled around, they are allowed to interact with other flux elements on the surface. When two elements approach each other to within a minimum separation distance (here set to $4.2 \mathrm{Mm}$ ), they are said to undergo a "collision", and are replaced by a single flux element having a flux equal to the sum of the flux possessed by the colliding elements. It is important to note that such collisions between flux elements with opposite polarities result in (partial) cancellations, are the only way in which flux is removed from the model photosphere.

\subsection{Properties of a Solar-Like Model}

In the model using the empirically derived solar parameters (hereafter referred to as Case SUN), flux is inserted and transported across the model photosphere using prescriptions that match measurements of solar flux emergence and subsequent evolution. This model reproduces the statistical behavior of the flux on the solar photosphere very well over the course of several 11-year sunspot cycles. 


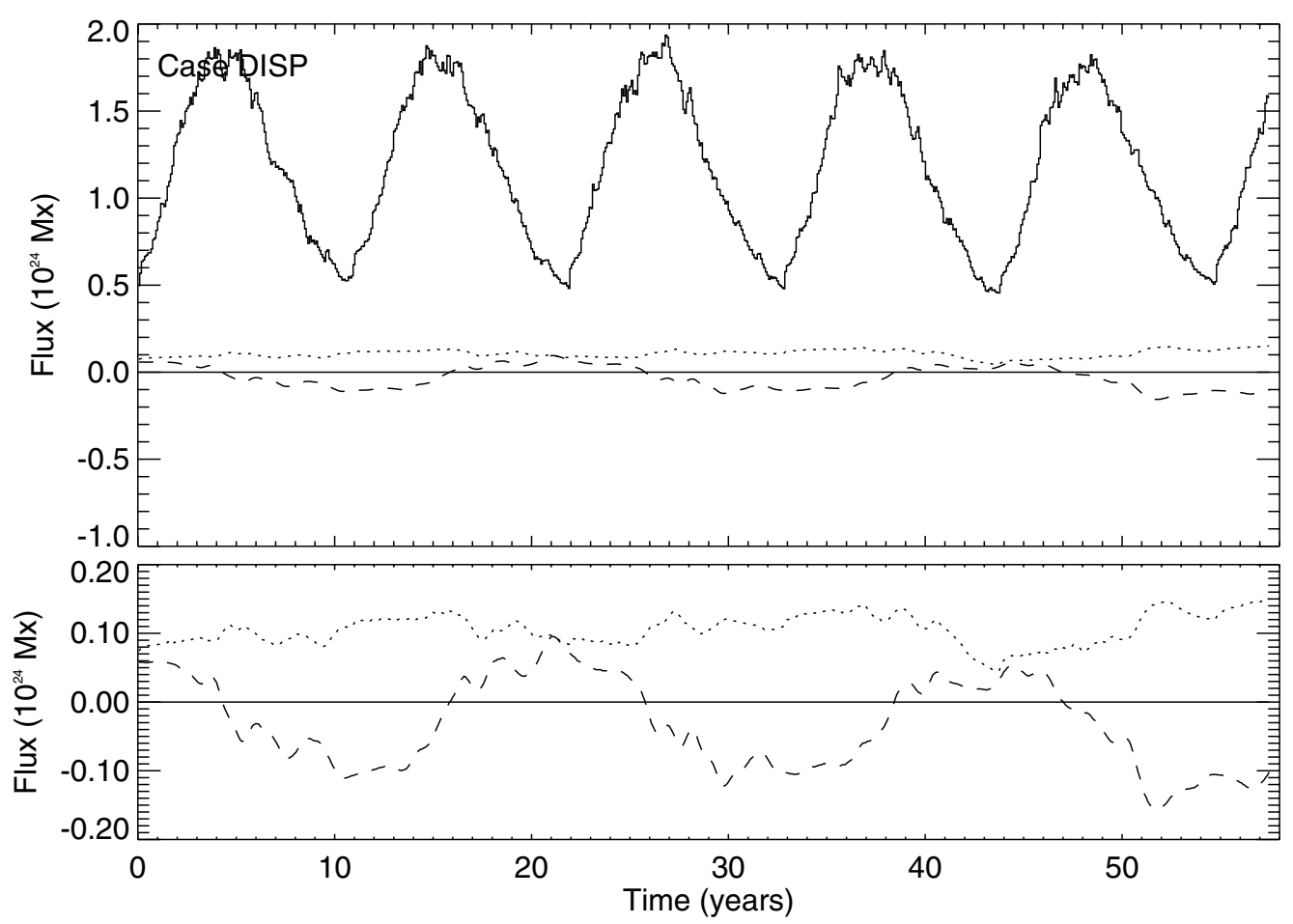

Figure 2. The total unsigned flux (solid line), total unsigned flux in the north polar cap (dotted line), and twice the net unsigned polar flux in the north polar cap (dashed line) for Case DISP. The north polar cap fluxes are evaluated using all flux north of $+60^{\circ}$ in latitude.

In Figure 1 is plotted the total unsigned flux on the model photosphere, and it follows the variation in large-scale flux emergence throughout each 11-year sunspot cycle. Also plotted is the amount of flux in the polar caps, defined here to be the regions poleward of $60^{\circ}$ latitude in each hemisphere. The polar-cap flux varies in antiphase with the solar cycle, with maximum polar-cap flux occurring at solar minimum, matching the behavior observed on the sun.

\subsection{Varying the Flux Dispersal Properties}

With this flux-transport model in hand, we can now investigate the effects caused by varying the characteristic parameters away from their solar values, and then using Case SUN as a benchmark for comparison. Here, we focus on how changes in the nature of flux dispersal affect the large-scale surface-flux patterns over the course of a sunspot cycle. Because the dispersal is governed by the most mobile (i.e., the smallest) flux elements, we are able to develop some intuition into the role that small-scale flux and its dispersal properties have on the formation of large-scale patterns such as the polar-cap fluxes and the associated poleward streaks.

For example, we ran a model with the same emergence characteristics as Case SUN, and with the same differential rotation and meridional flow profiles as Case SUN, but with the dispersal coefficient reduced by a factor of 10. Figure 2 shows the total unsigned, total unsigned north polar-cap, and net north polar-cap fluxes for this model (hereafter Case DISP). The unsigned flux in Case DISP, both in the polar caps and across the full spherical surface, is clearly greater than in Case SUN. This increased level of flux makes 
(a) Case SUN

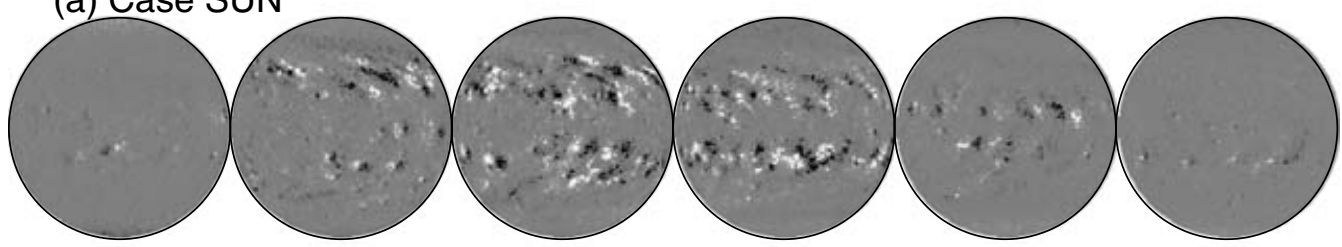

(b) Case LIN

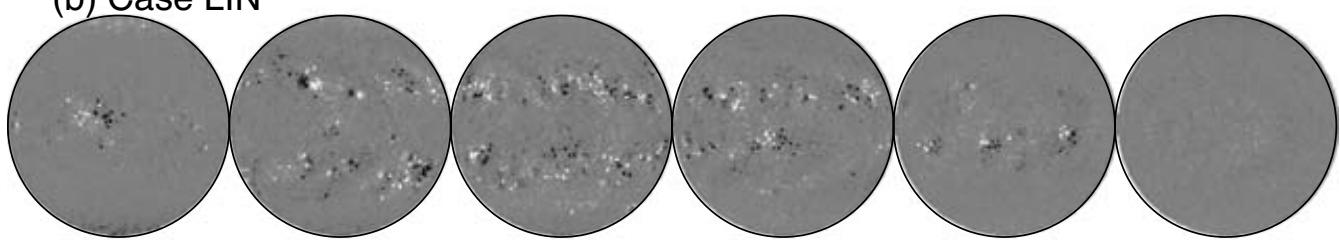

(c) Case DISP

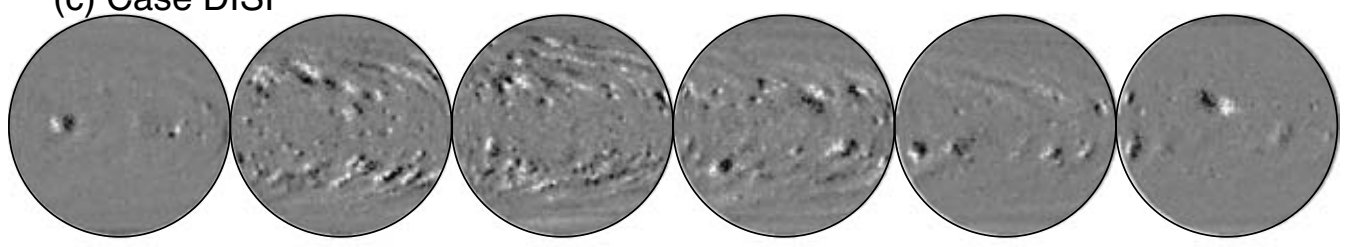

(d) Case LIN+DISP

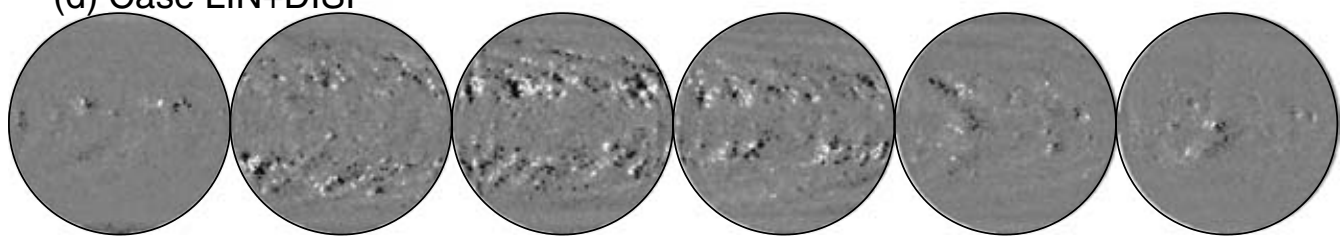

Figure 3. Equatorial views of Cases SUN, LIN, DISP, and LIN+DISP throughout half of a polarity (or a full sunspot) cycle, all scaled to $\pm 4 \times 10^{5} \mathrm{Mx} \mathrm{cm}^{-2}$. The first and last images in each row correspond to successive minima in the sunspot cycle.

sense when one considers that reducing dispersal coefficient results in fewer interactions between the flux elements, causing the number of cancellations to be lower, resulting in a greater amount of flux in the model.

\subsection{Large-Scale Patterns on the Disk Throughout a Sunspot Cycle}

We now introduce two additional cases to which the benchmark Case SUN can be compared. In Case LIN, the dependence of the mean free path length on flux has been removed, so that all magnetic flux elements in the model now disperse at the same rate regardless of the flux contained in each element. Essentially, the dispersal rate of smallscale flux has been reduced, and the dispersal rate of large-scale flux has been enhanced, relative to Case SUN. The last model, Case LIN+DISP, is a combination of Cases LIN and DISP.

Figure 3 shows the surface-flux patterns throughout a full sunspot cycle for all four cases. The consequences of the reduced cancellation rate in Case DISP can be seen in Figure 3c, in which the streaks of flux trailing away from the activity belt are more narrow and contain more flux than the corresponding features in Case SUN (Figure 3a).

Comparing Cases SUN and LIN in Figures 3a and 3b, we see that Case LIN now has much less flux on the disk than Case SUN. This effect results from of the now 
(a) Case SUN

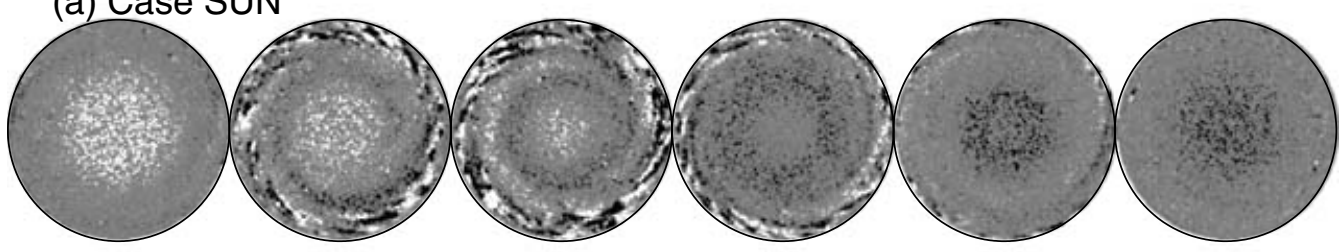

(b) Case LIN

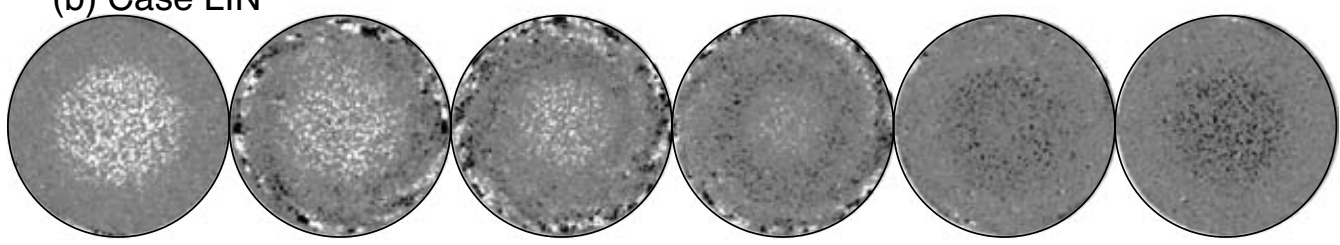

(c) Case DISP

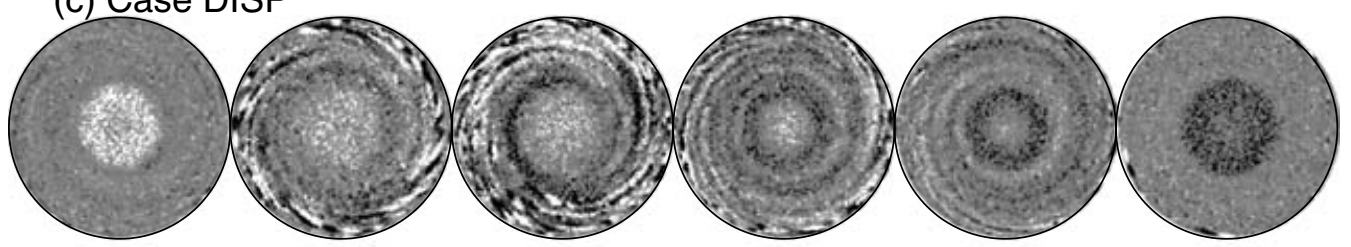

(d) Case LIN+DISP

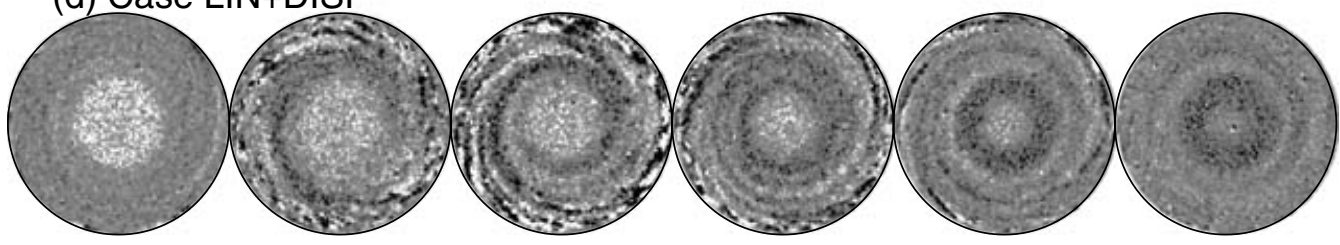

Figure 4. Polar views of Cases SUN, LIN, DISP, and LIN+DISP throughout half of a polarity (or a full sunspot) cycle, all scaled to $\pm 10^{5} \mathrm{Mx} \mathrm{cm}^{-2}$. The first and last images in each row correspond to successive minima in the sunspot cycle.

larger mobility of the large-scale flux concentrations, which in turn cause more flux to be cancelled before it can get dispersed into the background. Case LIN+DISP illustrates what happens when two competing and (partially) offsetting mechanisms are introduced into the model. As we have seen, a dispersal rate independent of flux results in an increase of the mean free path of the strongest flux elements, but this effect is somewhat counteracted by the reduced dispersal coefficient. In Case LIN, the amount of flux on the disk is about the same as in Case SUN, however the resulting patterns formed by this flux, such as the shapes of active region complexes, are noticeably different.

Figure 4 is similar to Figure 3, except that the viewpoint is now directly above the north pole, allowing the observer to see the patterns formed in the northern hemisphere in all four cases. In Figure 4a (Case SUN), one can clearly see the polar streaks, which now appear as spiral patterns from this perspective. The north polar cap during solar minimum takes the form of a prominent, but mildly diffuse, patch of unipolar flux centered at the north pole.

In Figure 4b (Case LIN), the polar cap is evidently about the same size and possesses the same the total flux as in Case SUN. When one looks more closely, however, one can see that the reversal from one polarity to the other is now different than in Case SUN. The mildly diffuse flux concentrations that comprise the polar cap are now less mobile 
than in Case SUN, causing the residual flux remaining from the previous sunspot cycle to remain visible for a longer period of time before its removal via cancellation.

In Figure 4c (Case DISP), the size of the polar cap is now smaller, and, owing to the increased amount of flux there (see Figure 2), therefore denser than in Case SUN. Our interpretation is that the size of the polar cap is determined by the competing effects of the poleward meridional flow (which tries to confine the flux at the pole) and the convective dispersal (which endeavors to spread the flux out). When the dispersal rate is decreased, as in Case DISP, the size of the polar cap becomes smaller.

Finally, Figure 4d (Case LIN+DISP) illustrates the relative importance of the two effects in determining the appearance of the polar caps. The size and appearance of the polar cap in Case LIN+DISP more closely resembles the polar cap in Case DISP than in Case LIN (although the polar cap is a bit more diffuse in Case LIN+DISP than in Case DISP), and thus we can infer that the dispersal rate plays the more prominent role. Additionally, the timing of the reversal in Case LIN+DISP is delayed slightly when compared with Case DISP, which is analogous to the differing reversals seen in Cases SUN and LIN.

\section{Concluding Remarks}

We have demonstrated that evolving surface-flux transport models can be used to investigate some aspects of the underlying mechanisms that cause the evolution of flux on the solar surface, without having to resort to solving the full magnetohydrodynamic equations. Such models are therefore useful for studying complex nonlinear dynamics of magnetism on the solar photosphere, and how these dynamical processes affect the long-term properties of the solar surface flux.

We conclude from these experiments that the small-scale flux on the sun is extremely important, due partly to their large numbers but mostly to their increased mobility relative to the large-scale flux concentrations. Because cancellation only occurs when flux elements of opposite polarity come into contact with each other, and because this process is the only way in which flux is removed from the model photosphere, these dynamics can play a critical role in the formation and evolution of the large-scale flux patterns evident in these models, and presumably too on the sun. Both the amount of flux that reaches the poles during each sunspot cycle, as well as the phasing and timing of the reversal of the polar-cap flux from cycle to cycle, are affected.

\section{References}

DeVore, C. R., Sheeley, Jr., N. R., \& Boris, J. P. 1984, Sol. Phys., 92, 1

Foukal, P., Harvey, K., \& Hill, F. 1991, ApJL, 383, 89

Hagenaar, H. J., Schrijver, C. J., \& Title, A. M. 2003, ApJ, 584, 1107

Hagenaar, H. J., Schrijver, C. J., Title, A. M., \& Shine, R. A. 1999, ApJ, 511, 932

Harvey, K. L. 1993, PhD thesis, University of Utrecht

Komm, R. W., Howard, R. F., \& Harvey, J. W. 1993a, Sol. Phys., 143, 19

-. 1993b, Sol. Phys., 147, 207

Leighton, R. B. 1969, ApJ, 156, 1

Muenier, N. 2003, A\&A, 405, 1107

Muller, R. \& Roudier, T. 1994, Sol. Phys., 152, 1131

Schrijver, C. J. 2001, ApJ, 547, 475

Schrijver, C. J., Shine, R. A., Hagenaar, H. J., Hurlburt, N. E., Title, A. M., Strous, L. H., Jefferies, S. M., Jones, A. R., Harvey, J. W., \& Duvall, Jr., T. L. 1996, ApJ, 468, 921

Sheeley, Jr., N. R., Nash, A. G., \& Wang, Y.-M. 1987, ApJ, 319, 481 